\title{
253 Anatomy of the palatine tonsil
}

A The crypts are lined by squamous epithelium.

B There are no afferent lymphatics.

C The tonsillar artery is a branch of the greater palatine.

D Pain sensation from the tonsil is carried in the glossopharyngeal nerve.

E The size of the tonsil can be assessed accurately by looking in the mouth while using a tongue depressor.

\section{The parapharyngeal space}

A Has no anatomical floor, allowing communication from skull base to superior mediastinum.

$B$ There is free communication with the retropharyngeal space.

C The deep lobe of the parotid projects into its lateral wall.

D Contents include the carotid sheath, the lower four cranial nerves, and deep cervical lymph nodes.

E At the level of C5 vertebra, the lateral wall is formed by the sternomastoid muscle. 